\title{
Tolerability of Tretinoin Lotion 0.05\% for Moderate to Severe Acne Vulgaris: A Post Hoc Analysis in a Black Population
}

\author{
Neal D. Bhatia, MD; William P. Werschler, MD; Fran E. Cook-Bolden, MD; \\ Eric Guenin, PharmD, PhD, MPH
}

\section{PRACTICE POINTS}

- Acne vulgaris is the most common dermatologic disorder seen in black patients, though data on treatment effects is lacking.

- Postinflammatory hyperpigmentation $(\mathrm{PIH})$ frequently coexists with acne, and retinoids are known to treat both.

- Tretinoin lotion $0.05 \%$ is effective in treating both inflammatory and noninflammatory lesions in black patients with acne and reducing $\mathrm{PIH}$ without the irritant contact dermatitis seen with other retinoid formulations.
Acne vulgaris (acne) is the most common dermatologic disorder seen in black patients. However, data are lacking on the effects of treatments, such as topical retinoids. Acne in black patients is frequently associated with postinflammatory hyperpigmentation $(\mathrm{PIH})$, which can be of greater concern than the patient's acne and often is the main reason these patients seek a dermatologist consultation. Retinoids can treat both acne and $\mathrm{PH}$. However, the potential for retinoids to induce an irritant contact dermatitis, which could lead to $\mathrm{PIH}$, is a concern. A lotion formulation of tretinoin was developed to provide an important alternative option to treat acne in black patients who may be sensitive to the irritant effects of other tretinoin formulations or where $\mathrm{PIH}$ is a concern.

Cutis. 2020;106:45-50.

A cne vulgaris (acne) is the most common dermatologic condition in black patients. ${ }^{1,2}$ However, among outpatient visits, racial disparities exist in both the likelihood of seeing a dermatologist and being treated. ${ }^{3}$ Black patients are less likely to visit a dermatologist or receive any acne medication. Acne in black skin is frequently associated with postinflammatory hyperpigmentation (PIH), an important consideration in treatment choice and maintenance.

There is a paucity of clinical studies that specifically evaluate acne treatment in this patient population. An 8-week, vehicle-controlled study with tretinoin cream $0.025 \%$ in 27 black patients with acne reported notable decreases in papules, pustules, and hyperpigmented macules in $83 \%$ of patients treated with tretinoin compared to only $13 \%$ receiving vehicle. ${ }^{4}$ However, irritation and inflammation were problematic. An open-label study of adapalene gel $0.1 \%$ in 65 black South Africans also demonstrated significant improvement in inflammatory and noninflammatory lesions and PIH $(P<.01)$, with seemingly better tolerability., $\mathrm{A}$ meta-analysis of 5 randomized studies from the United States and Europe $(\mathrm{N}=655)$ compared the efficacy and safety of adapalene gel $0.1 \%$ in black $(n=46)$ and white patients. ${ }^{7}$ There was no significant difference in percentage reduction in comedonal $(44 \%)$ or total $(42 \%)$ lesion counts. The percentage reduction in inflammatory lesion counts $(53 \%)$ was significantly greater in black patients $(P=.012)$. Tolerability also was better; black patients experienced significantly less erythema and scaling $(P<.001$ and $P=.026$, respectively), though erythema can be underestimated in darker skin tones because of the masking effects of melanin. ${ }^{5,7}$ Dryness was more common, though a smaller percentage

\footnotetext{
Dr. Bhatia is from Therapeutics Clinical Research, San Diego, California. Dr. Werschler is from Spokane Dermatology Clinic, Washington.

Dr. Cook-Bolden is from Mount Sinai Health Systems, New York, New York. Dr. Guenin is from Ortho Dermatologics, Bridgewater, New Jersey. Dr. Bhatia is a consultant for Ortho Dermatologics. Dr. Werschler is on the advisory board for and has received clinical trials support from Ortho Dermatologics. Dr. Cook-Bolden is a speaker for Ortho Dermatologics. Dr. Guenin is an employee of Ortho Dermatologics and holds an equity position in Bausch Health.

The eTable is available in the Appendix online at www.mdedge.com/dermatology.

Correspondence: Neal D. Bhatia, MD, Therapeutics Clinical Research, 9025 Balboa Ave, San Diego, CA 92123 (dsbconsulting37@gmail.com).
} 
of black patients reported moderate or severe dryness compared to white patients ( $7 \%$ vs $18 \%){ }^{7}$

Black patients also are less likely to receive combination therapy, and again clinical data are limited. ${ }^{3}$ A more recent subgroup analysis evaluated the safety and efficacy of adapalene $0.1 \%$-benzoyl peroxide $2.5 \%$ gel in black patients with moderate acne from 3 studies $\left(n=238\right.$ out of a total of 3855 patients). ${ }^{8}$ Similar results were obtained as in the overall study populations, with $64.3 \%$ and $48.5 \%$ reductions in inflammatory and noninflammatory lesion counts, respectively, at week 12 . The most common treatment-related adverse event (AE) in both treatment groups was dry skin (11.3\%). ${ }^{8}$

Extensive clinical data in a predominantly white population have shown that topical retinoids (eg, tretinoin, adapalene, tazarotene) are highly effective in treating acne, and they are recommended as the cornerstone of topical therapy. ${ }^{9}$ However, there is a common perception that they are primarily effective in comedonal acne $\mathrm{e}^{10}$ and that their use is associated with notable cutaneous irritation. ${ }^{11,12}$ Several attempts have been made to alleviate the tolerability issue using novel delivery systems. A new lotion formulation of tretinoin recently was developed and leveraged polymeric emulsion technology with the aim to improve both efficacy and tolerability of tretinoin. Herein, we performed a post hoc analysis of 2 large phase 3 clinical studies ${ }^{13}$ in patients with moderate or severe acne treated with tretinoin lotion $0.05 \%$ to evaluate its safety and tolerability in a black population.

\section{METHODS Study Design}

We conducted a post hoc analysis of 2 identical multicenter, randomized, double-blind, vehicle-controlled, parallel-group clinical studies ${ }^{13}$ in black patients with moderate or severe acne. Protocols received approval from the appropriate institutional review board for each center before patient enrollment, and the studies were conducted in accordance with the Declaration of Helsinki and Good Clinical Practice as well as in compliance with local regulatory requirements. All patients were informed of the study details and provided written consent before entering the studies.

Patients were enrolled with an evaluator global severity score (EGSS) of 3 (moderate) or 4 (severe). Participants were randomized (1:1) to receive tretinoin lotion $0.05 \%$ or vehicle applied to the face once daily for 12 weeks.

\section{Study Population}

Eligible patients for the post hoc analysis included male and female patients with black skin who were 9 years and older and presented with 20 to 40 inflammatory lesions (papules, pustules, and nodules), 20 to 100 noninflammatory lesions (open and closed comedones), and 2 or fewer nodules. A washout period of up to 1 month was required for patients who previously used prescription and over-thecounter acne treatments, and a washout period of 6 months was required for systemic retinoids.

\section{Safety Evaluation}

Cutaneous safety (erythema and scaling) and tolerability (itching, burning, and stinging) were evaluated on a 4 -point scale $(0=$ none; $3=$ severe $)$. Severity of hypopigmentation and hyperpigmentation also was assessed using this 4-point scale. The investigator assessed erythema and scaling at the time of each study visit. Reports of itching, burning, and stinging were solicited from participants and recorded as an average score of their symptoms during the period since the prior visit.

Adverse events were evaluated throughout and summarized by treatment group, severity, and relationship to study medication.

\section{Statistical Analysis}

The safety analysis set comprised all randomized patients who were presumed to have used the study drug at least once and who provided at least 1 postbaseline evaluation. All AEs occurring during the studies were recorded and coded using the Medical Dictionary for Regulatory Activities version 18.0. Treatment group comparisons were made by tabulating the frequency of participants reporting 1 or more AEs during the study.

Cutaneous safety (scaling, erythema, hypopigmentation, and hyperpigmentation) and tolerability (itching, burning, and stinging) scores were presented by treatment group with descriptive statistics at baseline and weeks 4,8 , and 12 . Frequencies and percentages for each outcome category were included in the statistics.

\section{RESULTS \\ Baseline Characteristics}

A total of 308 patients were included in the post hoc analysis. Overall, $257(83.4 \%)$ patients completed the studies, including $138(83.6 \%)$ patients receiving tretinoin lotion $0.05 \%$ and 119 (83.2\%) receiving vehicle (Figure 1). Completion rates were similar in the female and male subgroups ( $83.3 \%$ and $83.8 \%$, respectively). The most common reasons for study discontinuations were lost to follow-up ( $\mathrm{n}=32 ; 10.4 \%)$ or participant request $(n=13 ; 4.2 \%)$ and were similar irrespective of treatment or sex. There were no study discontinuations due to AEs.

Demographic data (Table) were similar across the 2 treatment arms. The mean age (standard deviation [SD]) of the participants was 22.1 (8.35) years (range, 9-58 years). Participants were predominantly female (209/308 [67.9\%]) and tended to be a little older than the males (mean age, 23.6 vs 18.8 years).

There were no noticeable differences between treatment groups regarding baseline lesion counts or EGSS. At baseline, the mean number (SD) of inflammatory and noninflammatory lesions was 25.2 (4.87) and 41.1 (16.55), 


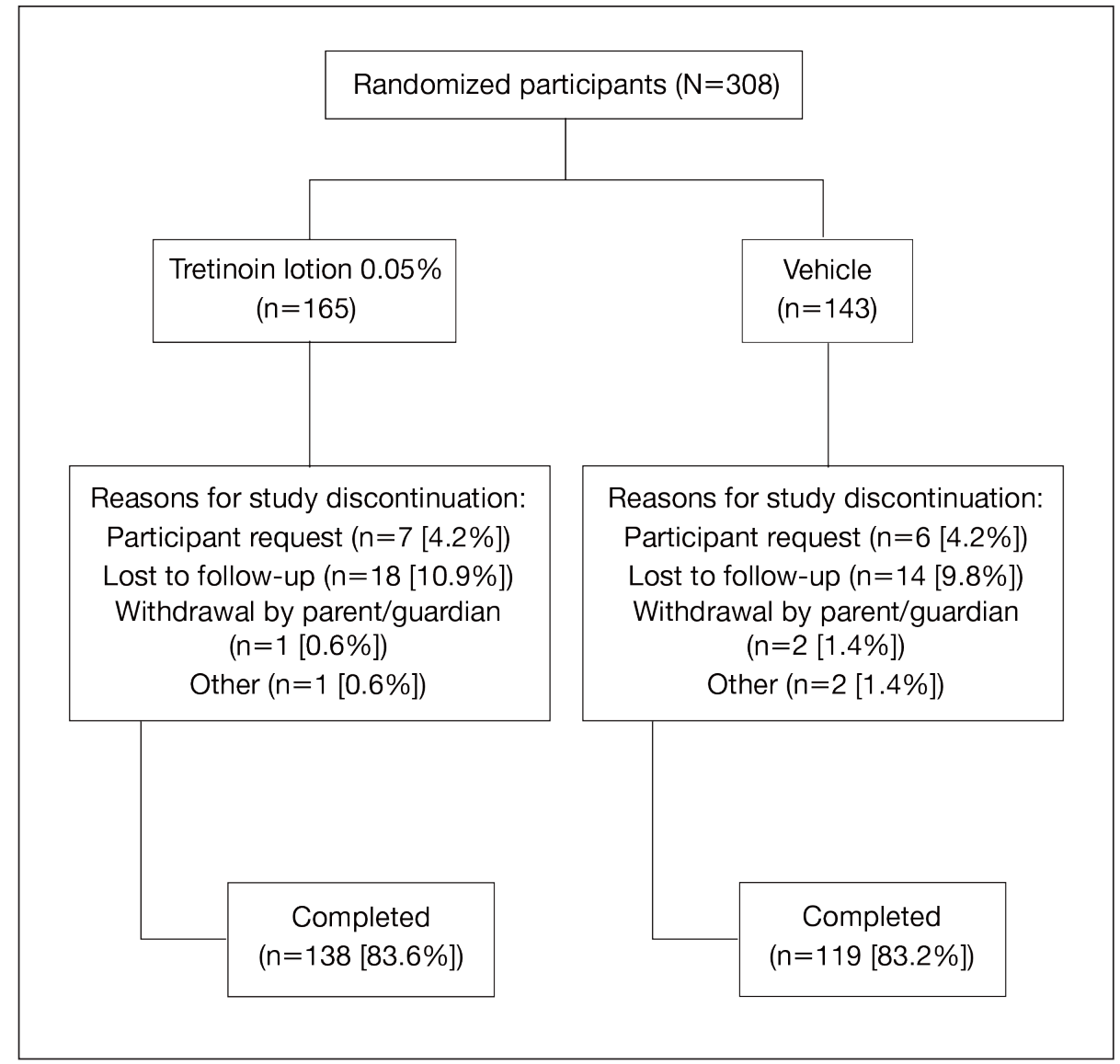

FIGURE 1. Participant disposition of black acne population showing percentage completion and reasons for discontinuation (intentionto-treat population; pooled data; $\mathrm{N}=308)$.

\section{Demographics and Baseline Characteristics for Black Acne Population Treated With

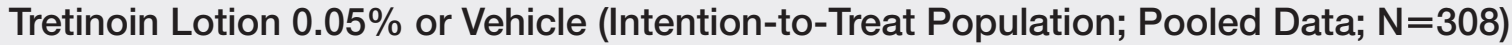

\begin{tabular}{|c|c|c|c|}
\hline & $\begin{array}{l}\text { Tretinoin Lotion } 0.05 \% \\
(n=165)\end{array}$ & $\begin{array}{l}\text { Vehicle } \\
(n=143)\end{array}$ & $\begin{array}{l}\text { Total } \\
(\mathrm{N}=308)\end{array}$ \\
\hline Mean age (SD), y & $21.9(7.96)$ & $22.3(8.81)$ & $22.1(8.35)$ \\
\hline Age range, y & $9-54$ & $10-58$ & $9-58$ \\
\hline \multicolumn{4}{|l|}{ Sex, n (\%) } \\
\hline Male & $54(32.7)$ & $45(31.5)$ & 99 (32.1) \\
\hline Female & $111(67.3)$ & $98(68.5)$ & $209(67.9)$ \\
\hline \multicolumn{4}{|l|}{ Ethnicity, n (\%) } \\
\hline Hispanic or Latino & $63(38.2)$ & $49(34.3)$ & $112(36.4)$ \\
\hline Not Hispanic or Latino & $102(61.8)$ & $94(65.7)$ & $196(63.6)$ \\
\hline \multicolumn{4}{|l|}{ Evaluator global severity score, $\mathrm{n}(\%)$} \\
\hline $3=$ moderate & $160(97.0)$ & $126(88.1)$ & $286(92.9)$ \\
\hline $4=$ severe & $5(3.0)$ & $17(11.9)$ & $22(7.1)$ \\
\hline Mean inflammatory lesion count (SD) & $25.2(4.89)$ & $25.2(4.87)$ & $25.2(4.87)$ \\
\hline Mean noninflammatory lesion count (SD) & $40.8(16.15)$ & $41.5(17.05)$ & $41.1(16.55)$ \\
\hline
\end{tabular}

Abbreviation: SD, standard deviation. 
respectively. At baseline, 286 (92.9\%) participants had moderate acne $(\mathrm{EGSS}=3)$. A higher proportion of male participants $(10.1 \%)$ had severe acne $(\mathrm{EGSS}=4)$ at baseline compared to female participants (5.7\%).

At baseline, the mean score (SD) for scaling, erythema, itching, burning, and stinging in those participants that were subsequently treated with tretinoin lotion $0.05 \%$ was 0.2 (0.42), 0.4 (0.68), 0.3 (0.60), 0.1 (0.28), and $0.1(0.32)$, respectively (where $1=$ mild)(Figure 2). There were no differences in mean baseline scores between active and vehicle treatment groups for hyperpigmentation ( 0.8 each) and hypopigmentation ( 0.1 each) in the active and vehicle treatment groups. Mean baseline scores were slightly higher in the female participants (0.9) compared to male participants (0.6). Baseline moderate or severe hyperpigmentation was reported in $23.2 \%$ and $3.2 \%$ of participants, respectively, who were subsequently treated with tretinoin lotion $0.05 \%$, which also was more commonly reported in female participants (33/105 [31.5\%]) than male participants $(8 / 50[16.0 \%])$.

\section{Safety}

Treatment-Related AEs-More participants treated with tretinoin lotion $0.05 \%$ reported treatment-emergent AEs (TEAEs) compared to vehicle (35 vs 18). The majority of participants reporting TEAEs were female (24 of 35). There were $2(1.3 \%)$ serious AEs with tretinoin lotion 0.05\% (both female), and 1 female participant $(0.6 \%)$ discontinued the study drug because of a TEAE (eTable).

Overall, there were $12(7.7 \%)$ treatment-related AEs; all were mild $(n=10)$ or moderate $(n=2)$. Treatmentrelated AEs reported by more than $1 \%$ of participants treated with tretinoin lotion $0.05 \%$ included applicationsite pain $(n=4 ; 2.6 \%)$, dryness $(n=4 ; 2.6 \%)$, irritation $(n=2 ; 1.3 \%)$, exfoliation $(n=2 ; 1.3 \%)$, or erythema $(n=2$; $1.3 \%)$. The majority of treatment-related AEs (10/12) were reported in the female subgroup. Although applicationsite pain $(3.4 \%)$ and dryness $(3.8 \%)$ were more commonly reported in the white population (unpublished data, Ortho Dermatologics) in the 2 studies, differences between the 2 racial groups were not significant.

Cutaneous Safety and Tolerability —Erythema and scaling were recorded by the investigator. Mild to moderate erythema was noted in $31 \%$ of participants at baseline, with $21 \%$ reporting mild to moderate scaling. Both improved over the study period following treatment with tretinoin lotion $0.05 \%$, with $79 \%$ of participants having no erythema and $88 \%$ having no scaling by week 12 . Mean scores for erythema and scaling remained less than 0.5 throughout the study $(1=$ mild $)$. There were slight transient increases in the mean baseline score for scaling (from 0.2 to 0.3 ) at week 4 in the active treatment group. By week 12, mean scores were half those reported at baseline (Figure 2).

Severity of itching, burning, and stinging was reported by participants. Overall, $23 \%$ reported mild to moderate itching at baseline. Only 7 participants $(5 \%)$ reported any itching by week 12 in the tretinoin lotion $0.05 \%$ group. Reports of burning and stinging were both rare and mild at baseline. Mean scores for itching, burning, and stinging at baseline for those participants who were subsequently treated with tretinoin lotion $0.05 \%$ were $0.3,0.1$, and 0.1 , respectively $(1=$ mild $)$. Itching severity reduced progressively with treatment. There were slight transient increases in mean scores for burning (from 0.1 to 0.2 ) and stinging (from 0.1 to 0.2 ) at week 4 , returning to baseline levels or below by week 12 .

Hyperpigmentation and HypopigmentationThere was a progressive improvement in baseline hyperpigmentation severity in participants treated with tretinoin lotion $0.05 \%$; mean scores reduced from 0.8 at baseline to 0.6 by week 12 (Figure 3), with a similar improvement in both sexes (Figure 4). Moderate
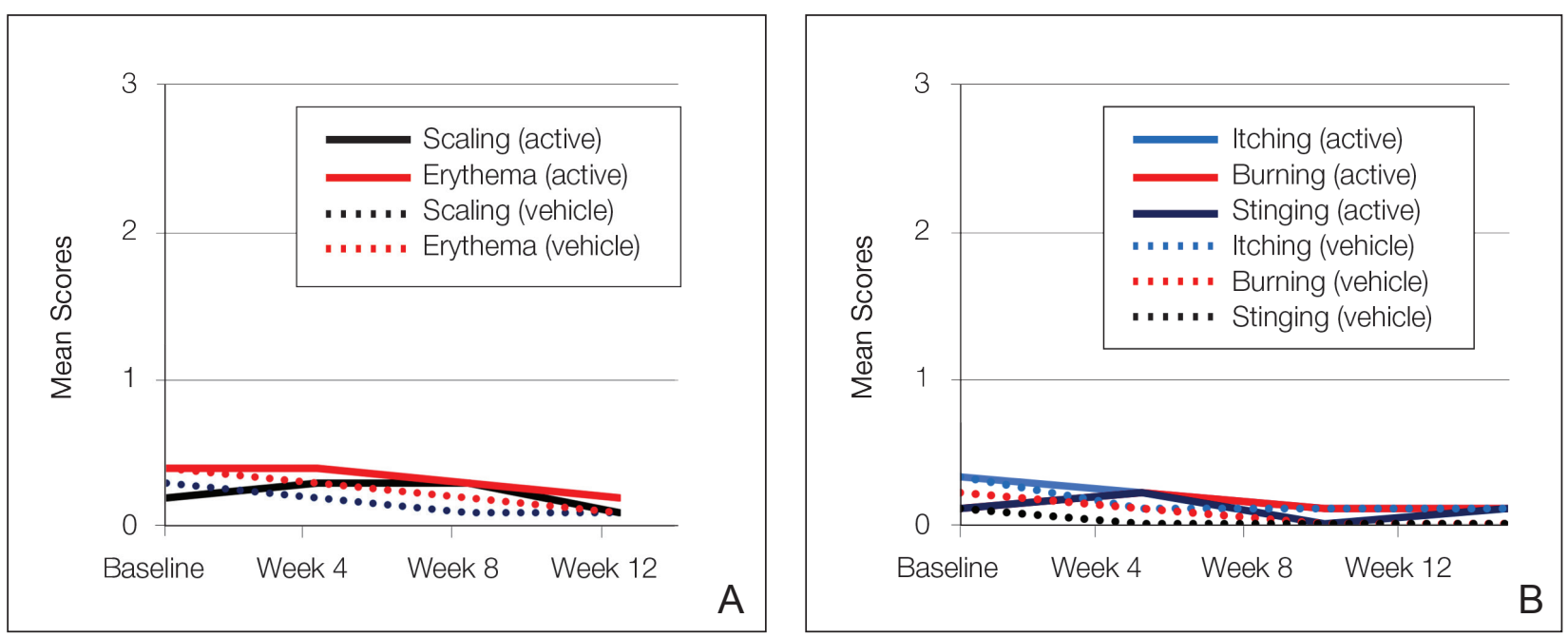

FIGURE 2. A and B, Cutaneous safety and tolerability assessment from baseline to week 12 for black population treated with tretinoin lotion $0.05 \%$ or vehicle (safety population; pooled data; $\mathrm{N}=291)$. Mean scores ranged from 0 to $3(0=$ none; $1=$ mild). 
to severe hyperpigmentation was reported in $24(17.3 \%)$ participants by week 12 compared to $41(26.4 \%)$ at baseline; the majority $(n=21)$ were female at week 12 . Moderate to severe hyperpigmentation was reported in $24(19.7 \%)$ participants treated with vehicle at week 12 .

Hypopigmentation at baseline was rare and mild, and again most common in female patients. There was no increase in hypopigmentation over the course of the study.

\section{COMMENT}

Topical retinoids (eg, tretinoin, adapalene, tazarotene) are recommended as the cornerstone of topical acne treatment, with safety and efficacy well documented in large pivotal trials. ${ }^{14}$ However, data in black patients are lacking. Acne is the most common dermatologic condition in these patients, and yet investigation into this important population is limited to small study populations or subgroup analyses.

Tretinoin lotion $0.05 \%$ is a novel topical treatment for moderate to severe acne that leverages polymeric emulsion technology. The development rationale was to provide a tretinoin formulation with improved efficacy and tolerability, features that could be especially suited to black patients with acne.

In our post hoc analysis of black patients with acne, tretinoin lotion $0.05 \%$ generally was considered safe and well tolerated. The most commonly reported treatment-related AEs were of low incidence and included applicationsite reactions and skin-related events attributed to the known properties of tretinoin. Most noteworthy was the extremely low irritation potential of this novel tretinoin formulation. Treatment-related AEs generally were mild, and interestingly, the majority occurred in female patients. The incidence of the most common treatment-related AEs-application-site dryness (2.6\%) and application-site pain $(2.6 \%$ ) -was lower than that reported in the white populations in the 2 studies (3.8\% and 3.4\%, respectively). (unpublished data, Ortho Dermatologics), though the differences were not significant $(P=.625$ and $P=.799)$.
Approximately one-quarter of participants had mild to moderate erythema, scaling, itching, and stinging at baseline. All of these cutaneous symptoms improved with treatment. There were slight transient increases in scaling and stinging at week 4 , with stinging more noticeable in the female population. There were no noticeable changes in mild to moderate burning during the study.

Postinflammatory hyperpigmentation is an important consideration in black patients with acne. It can arise from either acne-induced inflammation or injury. It can be of greater concern to the patient than the acne itself and often is the main reason black patients seek a dermatologist consultation. In a survey of adult female acne, nonwhite women experienced substantially more $\mathrm{PIH}$ than white women. In addition, clearance of PIH was most important for these nonwhite women ( $42 \%$ vs $8 \%$ for white women), whereas lesion clearance was the most important aspect for white women (58\% vs $32 \%$ for nonwhite women). ${ }^{15}$ Erring on the side of increased tolerability is appropriate in black patients with acne, given that any irritant reactions can lead to pigmentary alterations-hyperpigmentation or hypopigmentation - that can cause considerable patient anxiety. The psychologic impact of PIH can be devastating, and an ideal acne treatment in these patients would be one that is effective against both $\mathrm{PIH}$ and acne. Tretinoin cream $0.1 \%$ monotherapy has been shown to be effective in reducing $\mathrm{PIH} .{ }^{16}$ Postinflammatory hyperpigmentation lesions and normal skin were assessed by clinical and colorimetric evaluations and by analysis of biopsy specimens. Although facial PIH lesions in the 24 tretinoin-treated patients were significantly lighter after 40 weeks of treatment compared to vehicle in this study $(P<.001)$, overall improvement was first noted after 4 weeks $(P=.009)$. Normal skin also was minimally lightened by tretinoin; however, exuberant local skin reactions, including peeling, developed in $50 \%$ of patients. Mild to moderate $\mathrm{PIH}$ was present in the majority of tretinoin-treated patients at baseline in our post hoc analysis, severe in $3.2 \%$ of cases,

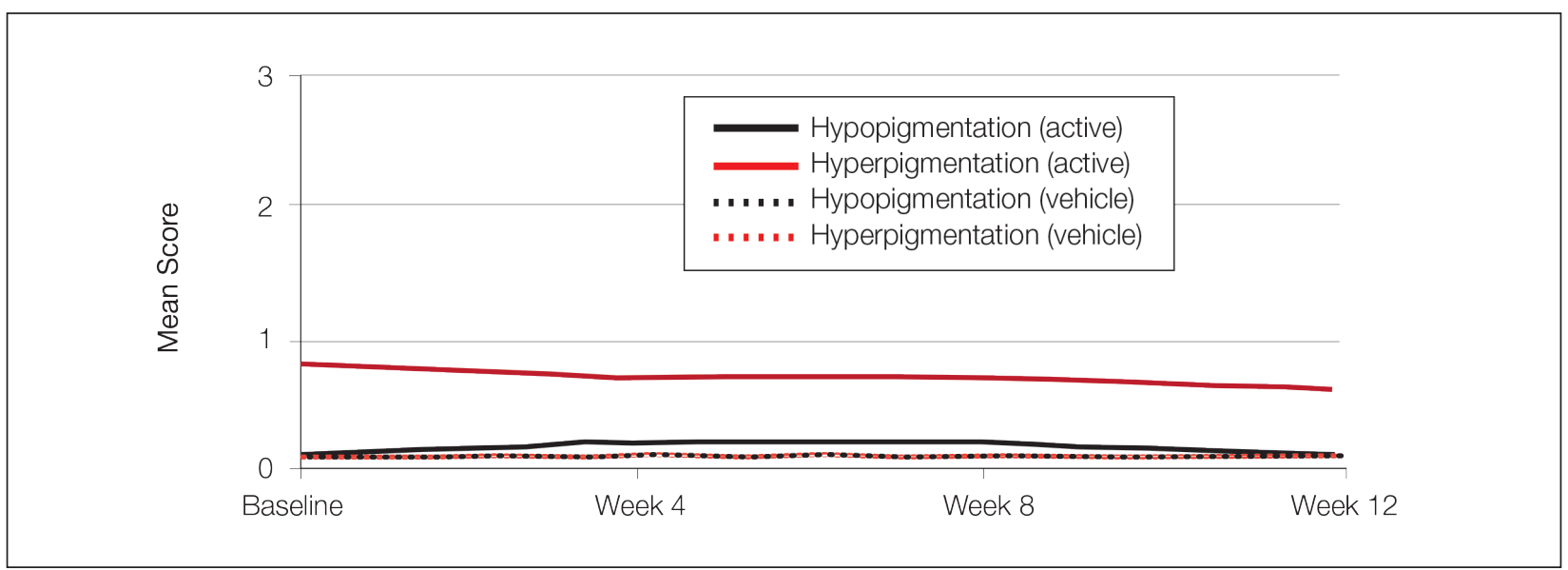

FIGURE 3. Postinflammatory hyperpigmentation and hypopigmentation in black patients treated with tretinoin lotion $0.05 \%$ or vehicle from baseline to week 12 (safety population; pooled data; $\mathrm{N}=291)$. Mean scores ranged from 0 to $3(0=$ none; $1=$ mild). 

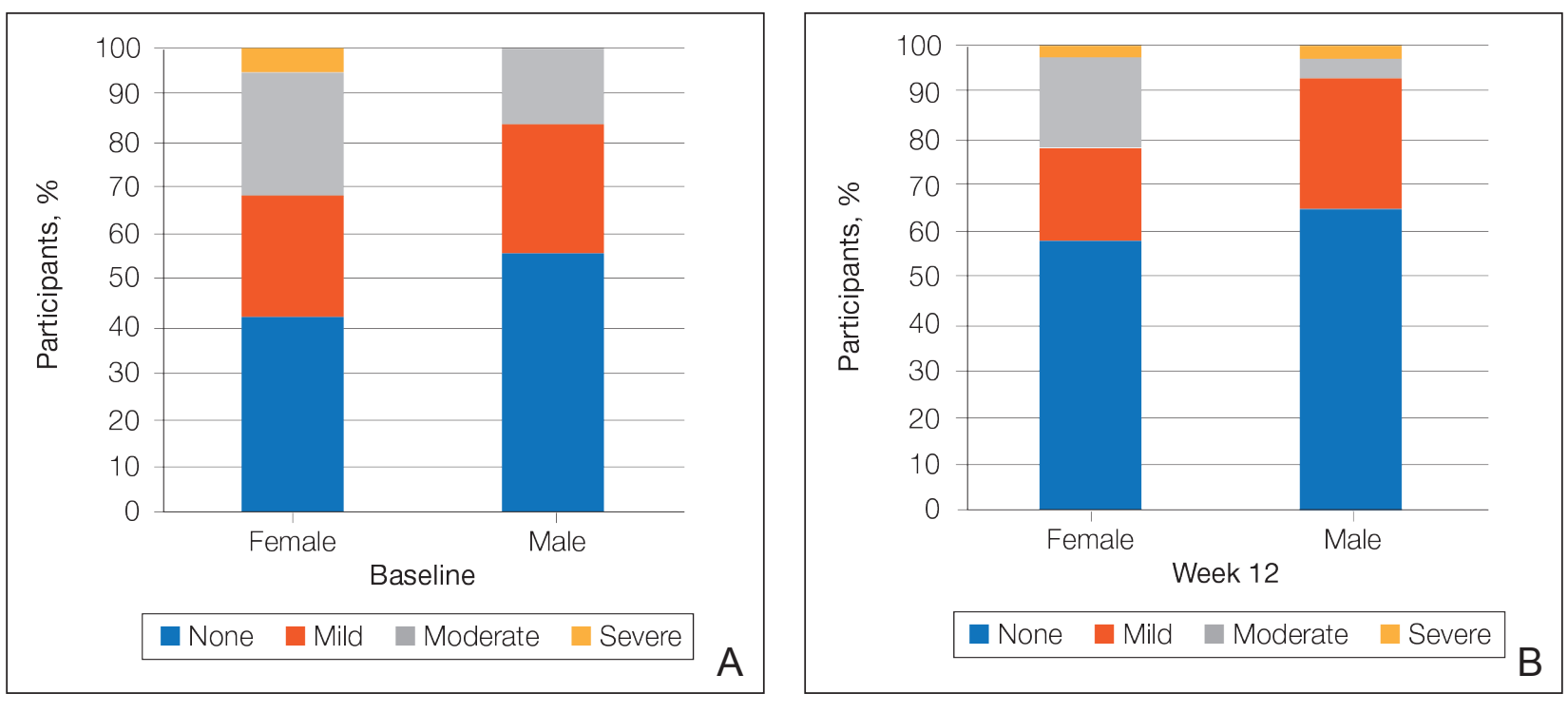

FIGURE 4. A and B, Postinflammatory hyperpigmentation severity at baseline and week 12 by sex. Severity was determined using 4-point scale $(0=$ none; 3 =severe)(safety population; male and female subgroups; $N=291$ ).

and both more common and severe in females. Mean scores reduced over the 12-week study period, from 0.6 to 0.4 in male patients and 0.9 to 0.7 in female patients. Hypopigmentation was rare and mild at baseline and did not increase over the course of the study. A pilot study with a cream formulation of tazarotene in patients with acne from darker racial groups showed the retinoid to be effective in treating PIH following 18 weeks of once-daily application. ${ }^{17}$ Further longer-term studies on treating PIH with tretinoin lotion $0.05 \%$ are warranted given its tolerability profile.

\section{CONCLUSION}

This novel tretinoin lotion $0.05 \%$ formulation is a safe and well-tolerated topical treatment for moderate to severe comedonal and inflammatory acne in black patients. Tretinoin lotion $0.05 \%$ does not appear to induce PIH and may afford an effective, well-tolerated, dual-treatment option.

Acknowledgments-We thank Brian Bulley, MSc (Konic Limited, United Kingdom), for medical writing support. Ortho Dermatologics funded Konic's activities pertaining to this manuscript.

\section{REFERENCES}

1. Halder RM, Grimes PE, McLaurin CI, et al. Incidence of common dermatoses in a predominantly black dermatologic practice. Cutis. 1983;32:388,390.

2. Taylor SC, Cook-Bolden F, Rahman Z, et al. Acne vulgaris in skin of color. J Am Acad Dermatol. 2002;46(suppl 2):S98-S106.

3. Rogers AT, SemenovYR, Kwatra SG, et al. Racial disparities in the management of acne: evidence from the National Ambulatory Medial Care Survey, 2005-2014. J Dermatolog Treat. 2018;29:287-289.
4. Halder RM. The role of retinoids in the management of cutaneous conditions in blacks. J Am Acad Dermatol. 1998;39(suppl 2):S98-S103.

5. Jacyk WK. Adapalene in the treatment of African patients. J Eur Acad Dermatol Venereol. 2001;15(suppl 3):37-42.

6. Jacyk WK, Mpofu P. Adapalene gel $0.1 \%$ for topical treatment of acne vulgaris in African patients. Cutis. 2001;68(suppl 4):48-54.

7. Czernielewski J, Poncet M, Mizzi F. Efficacy and cutaneous safety of adapalene in black patients versus white patients with acne vulgaris. Cutis. 2002;70:243-248.

8. Alexis AF, Johnson LA, Kerrouche N, et al. A subgroup analysis to evaluate the efficacy and safety of adapalene-benzoyl peroxide topical gel in black subjects with moderate acne. J Drugs Dermatol. 2014;13:170-174.

9. Leyden JJ, Shalita A, Thiboutot D, et al. Topical retinoids in inflammatory acne: a retrospective, investigator-blinded, vehicle-controlled, photographic assessment. Clin Ther. 2005;27:216-224.

10. Zaenglein AL, Pathy AL, Schlosser BJ, et al. Guidelines of care for the management of acne vulgaris. J Am Acad Dermatol. 2016;74:945-973.

11. Culp L, Moradi Tuchayi S, Alinia H, et al. Tolerability of topical retinoids: are there clinically meaningful differences among topical retinoids? J Cutan Med Surg. 2015;19:530-538.

12. Kircik LH. Evaluating tretinoin formulations in the treatment of acne. J Drugs Dermatol. 2014;13:466-470.

13. Tyring SK, Kircik LH, Pariser DM, et al. Novel tretinoin $0.05 \%$ lotion for the once-daily treatment of moderate-to-severe acne vulgaris: assessment of efficacy and safety in patients aged 9 years and older. J Drugs Dermatol. 2018;17:1084-1091.

14. Leyden J, Stein-Gold 1, Weiss J. Why topical retionoids are the mainstay of therapy for acne. Dermatol Ther (Heidelb) 2017;7:293-304.

15. Callender VD, Alexis AF, Daniels SR, et al. Racial differences in clinical characteristics, perceptions and behaviors, and psychosocial impact of adult female acne. J Clin Aesthet Dermatol. 2014;7:19-31.

16. Bulengo-Ransby SM, Griffiths CE, Kimbrough-Green CK, et al. Topical tretinoin (retinoic acid) therapy for hyperpigmented lesions caused by inflammation of the skin in black patients. $N$ Engl J Med. 1993;328:1438-1443.

17. Grimes P, Callender V. Tazarotene cream for postinflammatory hyperpigmentation and acne vulgaris in darker skin: a double-blind, randomized, vehicle-controlled study. Cutis. 2006;77:45-50. 


\section{APPENDIX}

eTABLE. Treatment-Emergent and Treatment-Related Adverse Events Through Week 12 for Black Acne Population Treated With Tretinoin Lotion $0.05 \%$ or Vehicle (Pooled Data; Safety Population; N=291)

\begin{tabular}{|c|c|c|}
\hline & \multicolumn{2}{|c|}{ No. of Participants (\%) } \\
\hline & Tretinoin Lotion $0.05 \%(n=155)$ & Vehicle Lotion $(n=136)$ \\
\hline Patients reporting any TEAE & $35(22.6)$ & $18(13.2)$ \\
\hline Patients reporting any serious $\mathrm{AE}$ & $2(1.3)$ & $0(0)$ \\
\hline Patients who died & $0(0)$ & $0(0)$ \\
\hline Patients who discontinued because of TEAE & $1(0.6)$ & $0(0)$ \\
\hline \multicolumn{3}{|l|}{ Severity of AEs reported } \\
\hline Mild & $25(16.1)$ & $14(10.3)$ \\
\hline Moderate & $10(6.5)$ & $3(2.2)$ \\
\hline Severe & $0(0)$ & $1(0.7)$ \\
\hline \multicolumn{3}{|l|}{ Relationship to study drug } \\
\hline Related & $12(7.7)$ & $2(1.5)$ \\
\hline Unrelated & $23(14.8)$ & $16(11.8)$ \\
\hline \multicolumn{3}{|c|}{ Treatment-related AEs reported by $\geq 1 \%$ of participants } \\
\hline Application-site pain & $4(2.6)$ & $1(0.7)$ \\
\hline Application-site dryness & $4(2.6)$ & $0(0)$ \\
\hline Application-site erythema & $2(1.3)$ & $1(0.7)$ \\
\hline Application-site exfoliation & $2(1.3)$ & $0(0)$ \\
\hline Application-site irritation & $2(1.3)$ & $0(0)$ \\
\hline
\end{tabular}

Abbreviations: TEAE, treatment-emergent adverse event; AE, adverse event. 